\section{Comparison of IOL power calculation formulae for pediatric eyes}

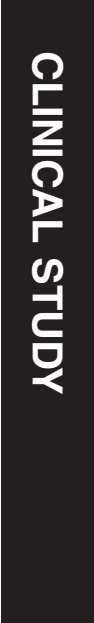

${ }^{1}$ lladevi Cataract \& IOL Research Centre, Raghudeep Eye Clinic, Ahmedabad, India

${ }^{2}$ Storm Eye Institute, Medical University of South Carolina, Charleston, SC, USA

Correspondence: $\checkmark$ Vasavada, lladevi Cataract \& IOL Research Centre, Raghudeep Eye Clinic, Gurukul Road, Memnagar, Ahmedabad 380052, India

Tel: +917927490909;

Fax: +917927411200

E-mail:vaishali@

raghudeepeyeclinic.com

Received: 31 March 2016 Accepted in revised form: 5 July 2016

Published online:

5 August 2016

\author{
Abstract \\ Purpose To evaluate and compare the \\ accuracy of modern intraocular lens (IOL) \\ power calculation formulae in pediatric eyes \\ and compare prediction error (PE) obtained \\ with manufacturer's vs personalized lens \\ constant. \\ Patients and methods An observational case \\ study was conducted in 117 eyes (117 patients) \\ undergoing pediatric cataract surgery with IOL \\ implantation. PE was calculated as predicted \\ refraction minus actual postoperative refraction, \\ and absolute $\mathrm{PE}$ as absolute difference \\ independent of the sign, $(\mathrm{APE})=$ predicted \\ refraction minus actual postoperative refrac- \\ tion. This was done for each formula using \\ manufacturer's and personalized lens constant. \\ Further, PE and APE were evaluated according \\ to axial length (AL). \\ Results Mean age of children was 2.97 years. \\ About 66/117 eyes (56.4\%) were below 2 years \\ of age. Using Holladay 2, Holladay 1, Hoffer \\ $\mathrm{Q}$, and SRK/T formulae with manufacturer's \\ lens constant, mean PE was 0.36, 0.41, 0.69, \\ and 0.28 diopter (D), respectively. With \\ personalized lens constant, it was $0.16,0.15$, \\ 0.50 , and $-0.12 \mathrm{D}$, respectively. Difference \\ in mean PE between the formulae was \\ statistically significant $(P<0.0001)$. SRK/T \\ and Holladay 2 formulae had the least PE, \\ both with manufacturer's and personalized \\ constant. For eyes with $\mathrm{AL}<20 \mathrm{~mm}, \mathrm{SRK} / \mathrm{T}$ \\ and Holladay 2 formulae gave the least PE. \\ Personalizing the lens constant led to a \\ decrease in mean PE in all formulae, \\ except the Hoffer $Q$ formula. However, \\ personalizing the lens constant did not \\ significantly improve the APE. At least 21\% \\ eyes had an APE of > 2 D with all formulae, \\ even with personalized lens constants. \\ Conclusion In pediatric eyes, SRK/T and \\ the Holladay 2 formulae had the least PE. \\ Personalizing the lens formula constant did \\ reduce the PE significantly for all formulae \\ except Hoffer Q. In extremely short eyes
}

V Vasavada1, SK Shah", VA Vasavada1, AR Vasavada', RH Trivedi ${ }^{2}$, S Srivastava ${ }^{1}$ and SA Vasavada ${ }^{1}$

$(\mathrm{AL}<20 \mathrm{~mm}), \mathrm{SRK} / \mathrm{T}$ and Holladay 2

formulae gave the best PE.

Eye (2016) 30, 1242-1250; doi:10.1038/eye.2016.171;

published online 5 August 2016

\section{Introduction}

Accurate determination of intraocular (IOL) power is one of the major challenges for the long-term care of children undergoing cataract surgery. Because all power calculation formulae have been derived from studies done on the adult eye, it is yet unclear which of these would give the best prediction of postoperative refractive error, especially with short axial length (AL) and high keratometry $(\mathrm{K})$ values and a target refraction that may be significantly different from plano. Further compounding this issue is the fact that these eyes are usually expected to change considerably in the postoperative years, resulting in significant refractive changes. ${ }^{1-3}$

Several studies on children have shown larger errors in IOL formula predictions than that are found in adults. ${ }^{4-9}$ Further, several authors have compared the accuracy of different IOL formulae designed for adult eyes in predicting early postoperative refractive error in eyes of infants and young children., ${ }^{7-12}$ However, there is no consensus as to which formula provides the best prediction of early postoperative refraction, particularly in the extremely small eye. In adult eyes, formulae that may have an advantage in terms of accuracy based on AL are: $\mathrm{AL}<22 \mathrm{~mm}$ : Hoffer Q, Holladay 2; AL 22-26 mm: Holladay I, Hoffer Q, SRK/T; AL > 26 mm: SRK/T,

Holladay $2 .{ }^{12}$ In pediatric eyes, however, results are inconsistent. ${ }^{4-8,10-12}$ Further, most published studies in the literature are retrospective. ${ }^{4-8,10,11}$

Optimizing or personalizing the lens constant has been recommended by authors of several formulae to improve their accuracy and predictability. ${ }^{13-16}$ Personalizing the lens constant can be used to make global adjustments for a variety of practice-specific variables (eg, surgical technique, 
variations in $\mathrm{AL}$ and $\mathrm{K}$ measurements, and so on). In fact, failure to personalize lens constants has been shown to give suboptimal refractive outcomes following cataract surgery in adult eyes.

The aim of this observational case study is to assess the PE (deviation from desired refraction) using Holladay 2, Holladay 1, Hoffer Q, and SRK-T IOL power calculation formulae in pediatric patients undergoing cataract surgery with primary IOL implantation. Further, we also compared PE when the manufacturer's lens constant for each formula was used ws when the optimized lens constant was used.

\section{Materials and methods}

This observational case study included children scheduled for cataract surgery with IOL implantation. Children younger than 15 years of age undergoing cataract surgery with primary implantation of an in-the-bag IOL were included. Eyes with traumatic cataract, subluxation of lens, scarring of cornea, active uveitis, previous intraocular surgery, preoperative IOP of $>25 \mathrm{~mm}$, and non-bag fixation of IOL were excluded. In patients with bilateral cataract, only one eye was included for the study. Patients were also excluded if reliable preoperative measurements of immersion AL, K, anterior chamber depth (ACD), lens thickness, or corneal diameter could not be obtained.

The study was approved by the Institutional Review Board. Informed consent was obtained from the parents/ legal guardians of the child prior to enrollment in the study. The study followed the tenets of the Declaration of Helsinki.

In all eyes, a bimanual lens aspiration followed by implantation of a single-piece hydrophobic acrylic IOL (Acrysof SN60WF or Acrysof SN60AT, Alcon Laboratories, Fortworth, TX, USA) in the capsular bag was performed by a single surgeon (ARV). A standardized surgical technique was used depending on the age of the patient. The power of the IOL for implantation was determined based on AL and K measurements, performed during examination under anesthesia wherever office measurements were not possible. A hand-held autorefractor keratometer (Nidek ARK 30, Nidek Co Ltd., Tokyo, Japan) was used to obtain K reading in operating room. The instrument is calibrated every month. At least two $\mathrm{K}$ measurements were taken. The two $\mathrm{K}$ readings were accepted only if they were within 1 diopter (D) of each other. If the two $\mathrm{K}$ readings were more than $1 \mathrm{D}$ apart, then a third measurement was taken and average of the two closest readings was used. A-scan biometry was performed using immersion technique (Ocuscan, Alcon Laboratories) by one of two experienced operators. Measurements from the scan that provided the best wave (ie highest peaks with a perpendicular retinal spike) were taken. The phakic setting on the ultrasound unit was used when obtaining the AL measurements. Calculations were made using an average of at least five readings with a SD of $<0.1 \mathrm{~mm}$. Horizontal corneal diameter was measured using calipers under the operating microscope. The visible horizontal white to white diameter was documented. The caliper had steps of $0.5 \mathrm{~mm}$.

The manufacturer IOL constant was used for power calculation. IOL power calculation was performed in all eyes using the SRK-T formula. The decision of how much emmetropic IOL power was to be under-corrected was based on age of the child at surgery, fellow eye status, and hereditary factors.

Postoperatively, all children were examined between 4 and 6 weeks, under anesthesia whenever required. A dilated retinoscopy was performed by a single examiner, and refined by subjective refraction in cooperative children. The examiner was masked to the predicted refraction. Wherever 10-0 nylon sutures were taken, suture removal was performed prior to performing the retinoscopy. When the child was examined under anesthesia, an interval of 10-15 min was allowed between suture removal and retinoscopy. Refraction was converted into spherical equivalent $(\mathrm{SE})(\mathrm{SE}=$ sphere $+1 / 2$ cylinder $)$ for analysis.

For the purpose of analysis, the eyes were divided into following subgroups depending on the AL:

- Short $\mathrm{AL}-<22.0 \mathrm{~mm}$. This subgroup was further subdivided as:

- Extremely short eyes (AL $<20 \mathrm{~mm}$ ), and

- Medium short eyes ( $\mathrm{AL} \geq 20 \mathrm{~mm}$ and $\leq 22 \mathrm{~mm}$ ).

- Average AL->22 mm and $<24.5 \mathrm{~mm}$.

- Long $\mathrm{AL} \longrightarrow \geq 24.5 \mathrm{~mm}$.

\section{Data collection}

In all eyes, the following data were collected and entered into an excel sheet (Microsoft, Microsoft Corporation, Redmond, WA, USA) for analysis: age at surgery, preoperative $\mathrm{AL}$, horizontal corneal diameter, $\mathrm{K}$ reading, IOL power implanted, IOL model implanted, postoperative refraction, and date of postoperative refraction.

Prediction error $(\mathrm{PE})=$ predicted refraction minus actual refraction. Here, the direction (sign) of the error was taken into consideration.

Absolute prediction error $(\mathrm{APE})=$ predicted refraction minus actual postoperative refraction. When APE was calculated, the sign or the direction of the error was not taken into consideration; only the difference in absolute 
values of predicted and actual postoperative refraction was considered.

\section{Personalization of lens constant}

Lens constant personalization was performed on the Holladay IOL consultant software for each of the two IOLs using preoperative data (excluding preoperative refraction) and postoperative refraction. In total, 30 eyes each of children implanted with Acrysof SN60WF (Alcon Laboratories) and Acrysof SN60AT (Alcon Laboratories) were selected from the Holladay IOL Consultant software database and personalized ACD was obtained for both, Acrysof SN60WF and Acrysof SN60AT IOLs. Based on this personalized ACD, an equivalent constant was obtained for each of the four formulae for each IOL design (SN60AT and SN60WF).

\section{Statistical analysis}

Statistical analysis was performed on the SPSS Software (SPSS 13, IBM Corporation, Armonk, NC, USA). Non-parametric tests (Wilcoxon signed rank test) were used to analyze whether there was a significant difference in the PE and APE between the four formulae (Holladay 2, Holladay 1, Hoffer Q, and SRK-T). This was done using the manufacturer's constant, as well as the personalized lens constant. Further, PE and APE were also analyzed for all the four formulae in subgroups based on AL. Number of eyes with an APE of $<1.0$ diopter (D) between 1 and $2 \mathrm{D}$, and $>2.0$ diopters were analyzed for manufacturer's lens constant as well as personalized lens constant. Difference in PE and APE was analyzed between the four formulae when using the manufacturer's constant vs the personalized lens constant.

\section{Results}

One-hundred thirty eyes of 130 patients were enrolled. However, in 10 eyes (10 children), immersion ultrasound AL could not be obtained, and they were therefore excluded from the analysis. In another three eyes, the IOL could not be placed in the capsular bag due to peripheral extension of anterior capsulorhexis and therefore they were excluded from analysis.

One-hundred seventeen eyes of 117 children were included in the analysis. The mean age of the children was 2.97 years. Table 1 shows the demographic and preoperative parameters of the patients. About $71 \%$ of the patients $(84 / 117)$ were below the age of 4 years. Sixty-six patients $(56.41 \%)$ were below the age of 2 years, of which 42 patients were below the age of 1 year. Only eight patients $(6.83 \%)$ were above the age of 8 years. Seventy-
Table 1 Baseline preoperative characteristics and postoperative refraction

\begin{tabular}{lrrr}
\hline Parameter & Mean \pm SD & Median & Range \\
\hline Age (years) & $2.97 \pm 2.97$ & 1.72 & $0.20-13.80$ \\
Axial length (mm) & $20.87 \pm 2.82$ & 20.68 & $17.11-26.12$ \\
K1 (D) & $44.01 \pm 2.24$ & 44.00 & $38.75-50.25$ \\
K2 (D) & $45.62 \pm 2.87$ & 45.50 & $39.34-56.50$ \\
Anterior chamber depth (mm) & $3.31 \pm 0.61$ & 3.41 & $1.54-4.54$ \\
Lens thickness (mm) & $3.94 \pm 0.96$ & 3.58 & $2.87-6.02$ \\
Corneal diameter (mm) & $10.93 \pm 0.82$ & 11.00 & $9.00-13.00$ \\
IOL power implanted & $23.03 \pm 4.44$ & 24.00 & $9.00-30.0$ \\
Follow-up duration (months) & $1.00 \pm 0.6$ & 0.88 & $0.50-1.70$ \\
Postoperative spherical & $3.64 \pm 2.67$ & 3.50 & $-0.75-10.00$ \\
equivalent (D) & & & \\
\hline
\end{tabular}

six eyes received an SN60WF IOL and 41 eyes received SN60AT IOLs (Alcon Laboratories).

Table 2a shows PE in all eyes, and in subgroups based on AL when using the manufacturer's lens constant. When all eyes were considered together, the least PE was obtained with the SRK/T, followed by the Holladay 2 formula. There was a significant difference in the mean PE between the four formulae $(P<0.0001$, Wilcoxon signed rank test). In the long-AL subgroup ( $\mathrm{AL}>24.5 \mathrm{~mm}$ ), there were only nine eyes, thereby precluding any meaningful analysis. In eyes with an AL $<20 \mathrm{~mm}$, the SRK-T and Holladay 2 formulae gave least PE, with the Holladay 1 formula close behind. In medium short eyes and average eyes, however, the Holladay 2 and Holladay 1 formulae were found to give least PE, followed by the SRK-T formula.

Table $2 \mathrm{~b}$ shows APE in all eyes, and in subgroups based on AL using the manufacturer's lens constant. When all eyes were analyzed together, there were no statistically significant differences between the four formulae. However, the SRK-T, Holladay 2, and Holladay 1 formulae were found to give lower APE. When looking at extremely short, medium short, and average eyes, all four formulae gave similar APE values, except for the Hoffer Q formula that gave a slightly higher APE in eyes smaller than $20 \mathrm{~mm}$.

Table 3 shows the personalized lens constants for each of the four formulae, for both IOLs. Using these personalized lens constants, PE and APE were calculated, as shown in Tables $4 a$ and $b$, respectively. The mean PE values reduced when using personalized lens constants. However, similar to when the manufacturer's constant was used, the SRK-T, Holladay 2, and Holladay 1 gave better results compared with the Hoffer $Q$ formula. The difference between the four formulae was significant when all 117 eyes were considered together $(P<0.001$, Wilcoxon signed rank test). 
Table 2a Prediction error in groups classified according to axial length using manufacturer's lens constant

\begin{tabular}{|c|c|c|c|c|c|c|}
\hline & Number of eyes (n) & Mean PE & $S D$ & Median & Minimum & Maximum \\
\hline \multicolumn{7}{|c|}{ Extremely short eyes $(<20 \mathrm{~mm})$} \\
\hline Holladay 1 & 41 & 1.19 & 2.10 & 1.24 & -2.7 & 6.00 \\
\hline Holladay 2 & 41 & 0.92 & 2.07 & 0.9 & -2.77 & 5.40 \\
\hline Hoffer Q & 41 & 1.88 & 2.17 & 1.93 & -2.52 & 6.92 \\
\hline SRK/T & 41 & 0.85 & 1.97 & 0.95 & -2.86 & 5.38 \\
\hline \multicolumn{7}{|c|}{ Medium short eyes $(\geq 20 \mathrm{~mm}$ to $<22 \mathrm{~mm}$ ) } \\
\hline Holladay 1 & 42 & -0.03 & 1.32 & 0.05 & -3.65 & 2.32 \\
\hline Holladay 2 & 42 & 0.01 & 1.25 & 0.1 & -3.24 & 2.55 \\
\hline Hoffer Q & 42 & 0.12 & 1.3 & 0.17 & -3.29 & 2.55 \\
\hline $\mathrm{SRK} / \mathrm{T}$ & 42 & -0.07 & 1.18 & -0.05 & -3.22 & 1.12 \\
\hline \multicolumn{7}{|c|}{ Average eyes ( $>22 \mathrm{~m}$ to $<24.5 \mathrm{~mm}$ ) } \\
\hline Holladay 1 & 25 & -0.36 & 0.95 & -0.35 & -2.06 & 1.96 \\
\hline Holladay 2 & 25 & -0.31 & 0.93 & -0.34 & -1.84 & 2.09 \\
\hline Hoffer Q & 25 & -0.38 & 0.95 & -0.41 & -2.02 & 1.92 \\
\hline $\mathrm{SRK} / \mathrm{T}$ & 25 & -0.41 & 1.00 & -0.29 & -2.16 & 1.16 \\
\hline \multicolumn{7}{|l|}{ All eyes ${ }^{\mathrm{a}}$} \\
\hline Holladay 1 & 117 & 0.41 & 1.76 & 0.15 & -3.65 & 6.00 \\
\hline Holladay 2 & 117 & 0.36 & 1.87 & 0.14 & -3.24 & 5.20 \\
\hline Hoffer Q & 117 & 0.69 & 2.30 & 0.21 & -3.29 & 6.92 \\
\hline SRK/T & 117 & 0.28 & 1.64 & 0.15 & -3.22 & 5.38 \\
\hline
\end{tabular}

${ }^{\text {a }}$-value $<0.0001$ (extremely significant), Wilcoxon signed rank test.

Table $\mathbf{2 b}$ Absolute prediction error in groups classified according to axial length using manufacturer's lens constant

\begin{tabular}{|c|c|c|c|c|c|c|}
\hline & Number of eyes (n) & Mean PE & $S D$ & Median & Minimum & Maximum \\
\hline \multicolumn{7}{|c|}{ Extremely short eyes } \\
\hline Holladay 1 & 41 & 1.89 & 1.47 & 1.40 & 0.14 & 6.00 \\
\hline Holladay 2 & 41 & 1.74 & 1.41 & 1.31 & 0.03 & 5.40 \\
\hline Hoffer Q & 41 & 2.33 & 1.65 & 1.95 & 0.07 & 6.92 \\
\hline SRK/T & 41 & 1.67 & 1.31 & 1.13 & 0.07 & 5.38 \\
\hline \multicolumn{7}{|c|}{ Medium short eyes } \\
\hline Holladay 1 & 42 & 0.99 & 0.84 & 0.75 & 0.01 & 3.65 \\
\hline Holladay 2 & 42 & 0.97 & 0.76 & 0.75 & 0.04 & 3.24 \\
\hline Hoffer Q & 42 & 1.01 & 0.80 & 0.86 & 0.01 & 3.29 \\
\hline $\mathrm{SRK} / \mathrm{T}$ & 42 & 0.90 & 0.75 & 0.70 & 0.01 & 3.22 \\
\hline \multicolumn{7}{|l|}{ Average eyes } \\
\hline Holladay 1 & 25 & 0.74 & 0.67 & 0.44 & 0.05 & 2.06 \\
\hline Holladay 2 & 25 & 0.73 & 0.63 & 0.43 & 0.11 & 2.09 \\
\hline Hoffer Q & 25 & 0.74 & 0.67 & 0.74 & 0.01 & 2.02 \\
\hline $\mathrm{SRK} / \mathrm{T}$ & 25 & 0.78 & 0.72 & 0.43 & 0.05 & 2.16 \\
\hline \multicolumn{7}{|l|}{ All eyes ${ }^{\mathrm{a}}$} \\
\hline Holladay 1 & 117 & 1.29 & 1.25 & 0.90 & 0.01 & 6.00 \\
\hline Holladay 2 & 117 & 1.23 & 1.18 & 0.91 & 0.02 & 5.40 \\
\hline Hoffer Q & 117 & 1.43 & 1.37 & 1.11 & 0.01 & 5.38 \\
\hline $\mathrm{SRK} / \mathrm{T}$ & 117 & 1.19 & 1.15 & 0.85 & 0.01 & 6.92 \\
\hline
\end{tabular}

${ }^{\mathrm{a}} P$-value $=0.163($ Wilcoxon signed rank test $)$.

The APE values were not statistically significantly different when the four formulae were compared in the entire group as a whole or when analyzing subgroups based on AL.
Figures $1 \mathrm{a}$ and $\mathrm{b}$ shows the difference in PE and APE when using the manufacturer's lens constant $v s$ personalized lens constant. Personalizing the lens constant reduced the PE significantly with all three 
formulae except the Hoffer Q formula (Figure 1a). However, personalizing the lens constant did not significantly reduce the APE for either formula (Figure 1b). In general, for all the four formulae, with manufacturer's lens constant and personalized lens constant, the mean PE was on the hyperopic side (undercorrection) in extremely short eyes, whereas it was on the myopic side (overcorrection) with medium short and average AL eyes.

An APE of $<1.0 \mathrm{D}$ was found in 53, 54.7, 47.9, and $56.4 \%$ eyes with the Holladay 2, Holladay 1 , Hoffer Q, and SRK/T formulae, respectively, when using the manufacturer's lens constant. Greater than

Table 3 Personalized lens constants for the different formulae

\begin{tabular}{llcc}
\hline & $\begin{array}{l}\text { Formula } \\
\text { constant }\end{array}$ & $\begin{array}{c}\text { Manufacturer } \\
\text { constant }\end{array}$ & $\begin{array}{c}\text { Equivalent } \\
\text { formula } \\
\text { constant }\end{array}$ \\
\hline SN60WF IOL & SF & 1.73 & 1.36 \\
Holladay I & ACD & 5.49 & 5.11 \\
Holladay II & ACD & 5.49 & 5.11 \\
Hoffer Q & A Constant & 118.89 & 118.24 \\
SRK/T & & & \\
SN60AT IOL & SF & 1.45 & 1.41 \\
Holladay I & ACD & 5.20 & 5.10 \\
Holladay II & ACD & 5.20 & 5.06 \\
Hoffer Q & A Constant & 118.4 & 118.6 \\
SRK/T & & & \\
\hline
\end{tabular}

two diopter APE was noted in $21.4 \%$ of eyes with the Holladay 2, Holladay 1, and SRK/T formulae and in $25 \%$ eyes with the Hoffer Q formula and manufacturer's lens constant. Even with lens constant personalization, at least $21 \%$ eyes had an APE of $>2.0 \mathrm{D}$ with each formula.

\section{Discussion}

We compared four commonly used theoretical IOL power calculation formulae in reference to their ability to predict refractive outcomes in pediatric IOL implantation, as they are reported to be more predictable than older formulae such as SRK and SRK II. ${ }^{14,17-21}$

Further, we also compared predictive accuracy of the four formulae when using manufacturer's vs personalized lens constants. The measured outcome was based on the actual refraction measured at $0.75-1.7$ months postoperatively $v$ s target refraction using tested formulae. We chose this time interval, as the eye has healed and long-term effects would be minimal at this time.

Our results show that all the four formulae were relatively inaccurate in predicting the refractions. However, the SRK/T formula had the least mean PE when all eyes were considered together, followed closely by the Holladay 2 formula. Also, we found that personalizing the lens constant led to a statistically significant reduction in the PE with all formulae except

Table 4a Prediction error in groups classified according to axial length using personalized lens constant

\begin{tabular}{|c|c|c|c|c|c|c|}
\hline & Number of eyes (n) & Mean PE & $S D$ & Median & Minimum & Maximum \\
\hline \multicolumn{7}{|c|}{ Extremely short eyes } \\
\hline Holladay 1 & 41 & 0.91 & 2.01 & 0.95 & -3.30 & 5.23 \\
\hline Holladay II & 41 & 0.75 & 2.00 & 0.78 & -3.08 & 4.87 \\
\hline Hoffer Q & 41 & 1.73 & 2.02 & 1.68 & -2.45 & 6.15 \\
\hline $\mathrm{SRK} / \mathrm{T}$ & 41 & 0.4 & 1.94 & 0.37 & -3.21 & 4.58 \\
\hline \multicolumn{7}{|c|}{ Medium short eyes } \\
\hline Holladay 1 & 42 & -0.37 & 1.34 & -0.23 & -4.37 & 2.05 \\
\hline Holladay 2 & 42 & -0.25 & 1.28 & -0.13 & -3.95 & 2.22 \\
\hline Hoffer Q & 42 & -0.15 & 1.32 & -0.06 & -4.03 & 2.17 \\
\hline $\mathrm{SRK} / \mathrm{T}$ & 42 & -0.54 & 1.18 & -0.48 & -3.95 & 1.8 \\
\hline \multicolumn{7}{|l|}{ Average eyes } \\
\hline Holladay 1 & 25 & -0.48 & 0.94 & -0.4 & -2.39 & 1.39 \\
\hline Holladay 2 & 25 & -0.41 & 0.92 & -0.33 & -2.27 & 1.51 \\
\hline Hoffer Q & 25 & -0.44 & 0.92 & -0.44 & -2.43 & 1.32 \\
\hline $\mathrm{SRK} / \mathrm{T}$ & 25 & -0.63 & 0.98 & -0.48 & -2.51 & 1.37 \\
\hline \multicolumn{7}{|l|}{ All eyes ${ }^{\mathrm{a}}$} \\
\hline Holladay 1 & 117 & 0.15 & 1.73 & -0.03 & -4.37 & 5.23 \\
\hline Holladay 2 & 117 & 0.16 & 1.67 & -0.04 & -3.95 & 5.03 \\
\hline Hoffer Q & 117 & 0.5 & 1.83 & 0.14 & -4.03 & 6.15 \\
\hline $\mathrm{SRK} / \mathrm{T}$ & 117 & -0.12 & 1.63 & -0.34 & -3.95 & 5.00 \\
\hline
\end{tabular}

${ }^{\mathrm{a}} P$-value $<0.0001$ (Wilcoxon signed rank test). 
Table $4 \mathrm{~b}$ Absolute prediction error in groups classified according to axial length using personalized lens constant

\begin{tabular}{|c|c|c|c|c|c|c|}
\hline & Number of eyes (n) & Mean PE & $S D$ & Median & Minimum & Maximum \\
\hline \multicolumn{7}{|c|}{ Extremely short eyes } \\
\hline Holladay 1 & 41 & 1.73 & 1.33 & 1.30 & 0.00 & 5.23 \\
\hline Holladay 2 & 41 & 1.66 & 1.31 & 1.19 & 0.10 & 4.87 \\
\hline Hoffer Q & 41 & 2.16 & 1.52 & 2.01 & 0.02 & 6.15 \\
\hline $\mathrm{SRK} / \mathrm{T}$ & 41 & 1.53 & 1.23 & 1.22 & 0.10 & 4.58 \\
\hline \multicolumn{7}{|c|}{ Medium short eyes } \\
\hline Holladay 1 & 42 & 1.03 & 0.92 & 0.80 & 0.02 & 4.37 \\
\hline Holladay 2 & 42 & 0.97 & 0.85 & 0.77 & 0.00 & 3.95 \\
\hline Hoffer Q & 42 & 1.00 & 0.85 & 0.86 & 0.01 & 4.03 \\
\hline $\mathrm{SRK} / \mathrm{T}$ & 42 & 0.99 & 0.82 & 0.76 & 0.01 & 3.95 \\
\hline \multicolumn{7}{|l|}{ Average eyes } \\
\hline Holladay 1 & 25 & 0.75 & 0.71 & 0.50 & 0.06 & 2.39 \\
\hline Holladay 2 & 25 & 0.73 & 0.66 & 0.41 & 0.10 & 2.27 \\
\hline Hoffer Q & 25 & 0.75 & 0.67 & 0.51 & 0.07 & 2.43 \\
\hline $\mathrm{SRK} / \mathrm{T}$ & 25 & 0.85 & 0.77 & 0.53 & 0.02 & 2.51 \\
\hline \multicolumn{7}{|l|}{ All eyes ${ }^{\mathrm{a}}$} \\
\hline Holladay 1 & 117 & 1.26 & 1.18 & 0.89 & 0.00 & 5.23 \\
\hline Holladay 2 & 117 & 1.20 & 1.15 & 0.87 & 0.00 & 5.03 \\
\hline Hoffer Q & 117 & 1.37 & 1.29 & 1.03 & 0.01 & 6.15 \\
\hline SRK/T & 117 & 1.19 & 1.10 & 0.82 & 0.01 & 5.00 \\
\hline
\end{tabular}

${ }^{\text {a }}$-value $=0.459$ (Wilcoxon signed rank test).

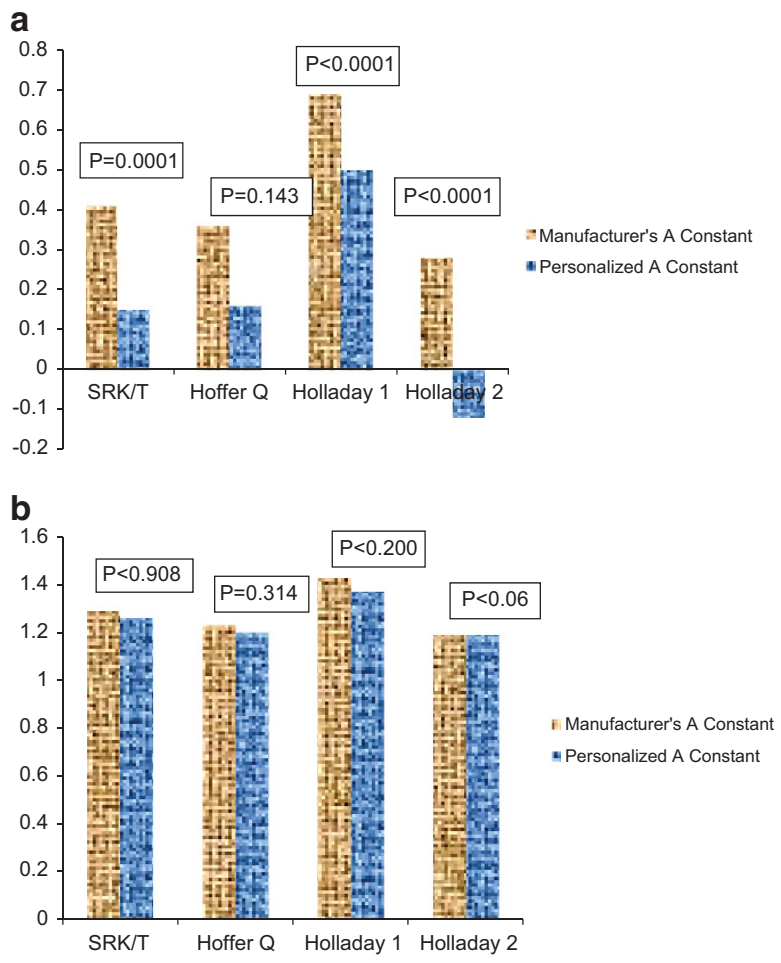

Figure 1 (a) Mean PE when using the manufacturer's lens constant $v$ s the personalized lens constant. There is a significant decrease in the PE in all formulae except Hoffer Q. (b) Mean APE when using the manufacturer's vs the personalized lens constant. There is no significant change in APE in all the formulae. the Hoffer Q formula, where although there was a decrease in PE (from 0.69 to $0.50 \mathrm{D}$ ), it did not attain statistical significance.

In the current study, there was a tendency for overcorrection (less residual hyperopia than expected) with all the formulae when using the manufacturer's lens constant. On using the personalized lens constant, this tendency for overcorrection decreased in all four formulae. Thus, personalizing the lens constant led to a more accurate prediction of the postoperative refraction. However, in young eyes with rapid and relatively unpredictable axial growth, as well as considering the fact that IOLs are only available in minimum steps of $0.5 \mathrm{D}$, it is not clear whether there will be any clinical impact of using personalized lens constants in these eyes.

Most published studies in the literature report that all IOL power calculation formulae are more or less qual in their predictive accuracy. ${ }^{4-9,11}$ Neely et $a l^{7}$ reported a mean PE of $0.3 \mathrm{D}(\mathrm{SD}-1.5 \mathrm{D})$. They also reported that among the formulae tested, that is, SRK II, SRK-T, Holladay 1, and Hoffer Q, there was no significant difference. In fact, the SRK II formula gave the least amount of variability overall, whereas the Hoffer Q generally gave the greatest amount of variability, particularly in eyes with an $\mathrm{AL}<19 \mathrm{~mm} .{ }^{4}$ Contrary to this, Nihalani and Vanderveen ${ }^{8}$ retrospectively studied 135 eyes and reported that the Hoffer Q formula was the most accurate in predicting postoperative refraction. 
The mean age of children in their series was 6.4 years, with 69 eyes having an AL of $<22 \mathrm{~mm}$. Andreo et al ${ }^{4}$ reported that there was minimal difference between SRK/T, SRK II, Holladay 1, and Hoffer Q formulae in terms of their predictive accuracy in short, medium, and long eyes. Vanderveen et al ${ }^{12}$ reported results from 43 eyes of infants, with a mean age of 2.5 months and $\mathrm{AL}<20 \mathrm{~mm}$ in most eyes. In this prospective, randomized multicenter trial, they reported that both SRK/T and Holladay 1 formulae worked equally well in terms of median prediction errors. Trivedi et $a l^{22}$ reported a comparison of the accuracy of Holladay 2 formula as compared with Holladay 1, Hoffer Q, and SRK/T formulae and they found that even in the absence of preoperative refraction, the Holladay 2 formula had the least PE and $\mathrm{APE}$, particularly so in short eyes (AL $<22.0 \mathrm{~mm})$. In a recently published study, Kekkunaya et al ${ }^{11}$ studied predictive accuracy of the SRK II, SRK/T, Holladay, and Hoffer $Q$ formulae in 128 eyes of children $<2$ years of age. They reported that SRK II formula was the most predictive, although all formulae had high APE. Further, they also reported that age, $\mathrm{AL}$, and $\mathrm{K}$ values did not influence predictive accuracy, except for the Holladay and Hoffer $Q$ formulae that were influenced by AL. On the other hand, several authors ${ }^{5,12}$ reported that a shorter AL had a significant bearing on the predictive accuracy of the IOL calculation formula.

Although mean PE tells us about the direction of miscalculation, the APE is a useful measure to compare overall predictive accuracy of the different formulae. The mean APE in our study was 1.23, 1.29, 1.43, and 1.19 D with the Holladay 2, Holladay 1, Hoffer Q, and SRK/T formulae, respectively, using the manufacturer's lens constant when all eyes were considered together. However, when eyes were subclassified according to $\mathrm{AL}$, the mean APE increased to 1.89, 1.74, 2.33, and $1.67 \mathrm{D}$, respectively, with the Holladay 1, Holladay 2, Hoffer Q, and SRK/T formulae in extremely short eyes $(\mathrm{AL}<20 \mathrm{~mm})$. Although personalization of the lens constant decreased the APE for all formulae, this difference was not statistically significant. One study ${ }^{11}$ has reported that age, $\mathrm{AL}$, and $\mathrm{K}$ values did not influence predictive accuracy, except for the Holladay and Hoffer Q formulae that were influenced by AL. On the other hand, several authors $5,10,12$ reported that a shorter AL had a significant bearing on the predictive accuracy of the IOL calculation formula.

In our series, the mean age of children was 2.96 years, with median age being 1.72 years. Further, the mean AL was $20.87 \mathrm{~mm}$ (range 17.11-26.12 $\mathrm{mm}$ ), and 83 of the 117 eyes $(71 \%)$ had an $\mathrm{AL}$ of $\leq 22 \mathrm{~mm}$. Thus, our results are similar to many other published pediatric studies, which have also reported greater APE in eyes with shorter AL. $5,8,11,12$
There is only one study in the literature ${ }^{12}$ comparing the effect of personalizing the lens constant on the predictive accuracy of the IOL calculation formula. However, this is a very specialized subset of infant eyes below the age of 7 months with unilateral cataracts. In adult eyes, it is known that even with state-of-the-art techniques and equipment, a surgeon who fails to optimize his constants could have less accurate outcomes than someone using older equipment who does optimize. ${ }^{22}$ Trivedi et al ${ }^{10}$ (Poster presentation at the annual meeting of the American Academy of Ophthalmology, 2011) reported that personalizing the lens constant improves the PE and $\mathrm{APE}$ in pediatric eyes. Whereas we found that the mean PE improved significantly after personalizing the lens constants. In our study, there was no significant reduction in the APE when using the personalized lens constants. One of the reasons could be that our mean age and AL were lower than the ones reported in this study.

Our study has significant limitations. The surgical technique was not identical in all eyes. Age-appropriate surgical strategy was used, which included performing a primary posterior capsulorhexis (PCCC) along with anterior vitrectomy in children younger than 3 years, only PCCC without anterior vitrectomy in children aged 3-6 years, and no PCCC in children older than 6 years. This may be a contributing factor in the effective lens position. But given the many nuances of the problem of pediatric cataracts, it may not be able to obtain a strictly defined cohort as in adult eyes. In our study, there were $<15$ children where no PCCC was performed. It would be interesting to study the prediction errors with different formulae in pediatric eyes with different strategies of managing the posterior capsule. The Holladay 2 formula requires input of seven preoperative variables in order to calculate the IOL power, including the preoperative refraction. In our study, we did not have preoperative refraction data. However, with a dense cataract in children, it is often very difficult to measure the preoperative refraction. Similar to a previous report, 10 we found that the Holladay 2 formula can be used in pediatric eyes despite the absence of preoperative refraction.

The Haigis-L formula with optimization of three constants (a0, a1, and a2) is another modern formula that is reported to be very accurate in adult eyes, including extremes of AL. However, personalization of these constants requires input of at least 200 eyes. We therefore did not include this formula for comparison of predictive accuracy in our study.

In summary, our study results indicate that while all the four formulae were relatively inaccurate in predicting early postoperative refraction, the SRK/T and Holladay 2 formulae had the least PE. This was 
particularly true in eyes having an AL of $<20 \mathrm{~mm}$. Also, personalizing the lens constant for each formula did improve the PE, although it did not significantly reduce the APE. These results may help the surgeon in making a choice of the preferred IOL calculation formula, or even modify their calculated IOL power in these small eyes. We suggest using either the SRK/T or the Holladay 2 formula for children. Further, the personalized lens constants reported in the current study may be useful reference values that may be used during IOL power calculations to improve the accuracy of prediction. ${ }^{23}$

\section{Summary}

\section{What was known before}

- Pediatric IOL power calculation is a challenge, and both younger age as well as smaller axial length are factors that make the prediction of postoperative refraction even more difficult. Although most modern formulae are pretty accurate in adult eyes, in pediatric eyes there is still no consensus as to which IOL formula gives the best results in terms of its predictive accuracy. Further, in adult eyes, it is well known that optimizing the lens constant for every surgeon helps to achieve better refractive results. However, there are very few studies looking at the predictive accuracy of modern formulae in eyes of children younger than 2 years of age, and comparing prediction errors after optimizing the lens constant $v$ s without optimizing it.

\section{What this study adds}

- In children younger than 2 years of age, the SRK/T and the Holladay 2 formulae gives the least prediction error, followed by the Holladay 1, and then the Hoffer Q formula. Further, when we personalized the lens constants for each formula, the tendency to undercorrect (more residual hyperopia than expected) reduced in SRK/T, Holladay 2, and Holladay 1, but not the Hoffer Q formula. However, despite lens constant personalization, there was no significant difference in the absolute prediction error (APE) in all formulae. Despite personalizing the lens constant, $22 \%$ eyes with all four tested formulae had an APE of $>2$ diopters. Therefore, in very small eyes, personalization of the lens constant may not improve predictive accuracy of formulae drastically. Overall, however, it would be fair to recommend both SRK/T and Holladay 2 formulae for IOL power calculation in children younger than 2 years of age.

\section{Conflict of interest}

The authors declare no conflict of interest.

\section{Acknowledgements}

Dr Abhay R Vasavada receives occasional research support grant from Alcon Laboratories, USA.

\section{References}

1 Plager DA, Kipfer H, Sprunger DT, Sondhi N, Neely D. Refractive change in pediatric pseudophakia: 6-year follow-up. J Cataract Refract Surg 2002; 28: 810-815.

2 Hutchinson AK, Wilson ME, Saunders RA. Outcomes and ocular growth rates after intraocular lens implantation in the first 2 years of life. J Cataract Refract Surg 1998; 24: 846-852.

3 Enyedi LB, Peterseim MW, Freedman SF, Buckley EG. Refractive changes after pediatric intraocular lens implantation. Am J Ophthalmol 1998; 126: 772-781.

4 Andreo LK, Wilson ME, Saunders RA. Predictive value of regression and theoretical IOLformulas in pediatric intraocular lens implantation. J Pediatr Ophthalmol Strabismus 1997; 34(4): 240-243.

5 Tromans C, Haigh PM, Biswas S, Lloyd IC. Accuracy of intraocular lens power calculation in paediatric cataract surgery. Br J Ophthalmol 2001; 85(8): 939-941.

6 Mezer E, Rootman DS, Abdolell M, Levin AV. Early postoperative refractive outcomes of pediatric intraocular lens implantation. J Cataract Refract Surg 2004; 30(3): 603-610.

7 Neely DE, Plager DA, Borger SM, Golub RL. Accuracy of intraocular lens calculations in infants and children undergoing cataract surgery. J AAPOS 2005; 9(2): 160-165.

8 Nihalani BR, VanderVeen DK. Comparison of intraocular lens power calculation formulae in pediatric eyes. Ophthalmology 2010; 117(8):1493-1499.

9 Eibschitz-Tsimhoni M, Archer SM, Del Monte MA. Intraocular lens power calculation in children. Surv Ophthalmol 2007; 52(5): 474-482.

10 Trivedi RH, Wilson ME, Reardon W. Accuracy of the Holladay 2 intraocular lens for pediatric eyes in the absence of preoperative refraction. J Cataract Refract Surg 2011; 37: 1239-1243.

11 Kekkunaya R, Gupta A, Sachdeva V, Rao HL, Vaddavalli PK, Prakash VO. Accuracy of intraocular lens power calculation formulae in children less than two years. Am J Ophthalmol 2012; 154: 13-19.

12 Vanderveen DK, Trivedi RH, Nizam A, Lynn MJ, Lamber SR. Infant Aphakia Treatment Study Group Predictability of intraocular lens power calculation formulae in infantile eyes with unilateral congenital cataract: results from the Infant Aphakia Treatment Study. Am J Ophthalmol 2013; 156: 1252-1260.

13 Prager TC, Hardten DR, Fogal BJ. Enhancing intraocular lens outcome precision: an evaluation of axial length determinations, keratometry, and IOL formulas. Ophthalmol Clin North Am 2006; 19(4): 435-448.

14 Hoffer KJ. The Hoffer Q formula: a comparison of theoretical and regression formulas. J Cataract Refract Surg 1993; 19: 700-712.

15 Holladay JT. Standardizing constants for ultrasonic biometry, keratometry and intraocular lens power calculations. J Cataract Refract Surg 1997; 23: 1356-1370.

16 Petermeier K, Gekeler F, Messias A, Spitzer MS, Haigis W, Szurman P. Intraocular lens power calculations and optimized constants for highly myopic eyes. J Cataract Refract Surg 2009; 35: 1575-1581.

17 Retzlaff JA, Sanders DR, Kraff MC. Development of the SRK/T intraocular lens implant power calculation formula. J Cataract Refract Surg 1990; 16: 333-340.

18 Barrett GD. An improved universal theoretical formula for improved intraocular lens power prediction. J Cataract Refract Surg 1993; 19: 713-720. 
19 Richards SC, Steen DW. Clinical evaluation of the Holladay and SRK-II formulas. J Cataract Refract Surg 1990; 16: 71-74.

20 Sanders DR, Retzlaff JA, Kraff MC, Gimbel HV, Raanan MG. Comparison of the SRK/T formula and other theoretical and regression formulas. J Cataract Refract Surg 1990; 16: 341-346.

21 Olson T, Thim K, Corydon L. Accuracy of the newer generation intraocular lens power calculation formulas in long and short eyes. J Cataract Refract Surg 1991; 17: 187-193.
22 Trivedi RH, Wilson ME, Reardon W. Accuracy of the Holladay 2 intraocular lens formula for pediatric eyes in the absence of preoperative refraction. J Cataract Refract Surg 2011; 37: 1239-1243.

23 Madge SN, Khong CH, Lamont M, Bansal A, Antcliff RJ. Optimization of biometry for IOL implantation using the Zeiss IOL Master. Acta Ophthalmol Scand 2005; 83: 436-438. 